\title{
PREDICTORS OF MORTALITY IN PATIENTS WITH ACUTE RENAL FAILURE
}

\author{
Mehmet Tugrul Sezer ${ }^{1}$, Murat Demir ${ }^{1}$, Gokhan Gungor ${ }^{2}$, Altug Senol \\ Suleyman Demirel University School of Medicine, Isparta, Turkey: Division of Nephrology, Department of Internal \\ Medicine $^{1}$; Department of Internal Medicine ${ }^{2}$
}

Summary: Mortality associated with acute renal failure (ARF) remains high despite of developments in therapy strategies and definition of different prognostic factors. Therefore, this study focused on to define new prognostic factors and especially regional characteristics of the ARF patients. One hundred fifteen ARF patients, diagnosed from November 1998 to May 2003, were included to this prospective and observational study. Clinical features, laboratory parameters, Acute Physiology and Chronic Health Evaluation (APACHE) III scores and co-morbid conditions of the patients were examined. Clinical and laboratory data, and APACHE III scores were recorded at the first nephrology consult day. Thirty of the patients (26\%) died. APACHE III scores, presence and the total number of co-morbid conditions and serum albumin levels at the time of first nephrology consultation were found as independent predictors of mortality. There was a negative correlation between APACHE III scores and serum albumin levels. Not only increased APACHE III score and presence of co-morbid conditions but also low serum albumin level was found as the predictors of mortality. However, only serum albumin level is seen as modifiable prognostic factor among these parameters. Therefore, further studies are necessary to determine the causes of hypoalbuminemia in patients with ARF and the effect of it's effective treatment on patients outcome.

Key words: Acute renal failure; Apache III score; Hypoalbuminemia; Mortality

\section{Introduction}

Acute renal failure (ARF) is an important clinical syndrome, characterized by a rapid decline in renal function and with high mortality rate. Mortality associated with ARF remains high despite recent advances in critical care medicine, renal replacement therapy and nutritional support. Numerous prognostic factors; oliguria, presence of diabetes mellitus, Acute Physiology and Chronic Health Evaluation (APACHE) III score and hypoalbuminemia had been defined recently $(2,3,4,7,13,16,20,25)$.

Because ARF is a heterogeneous syndrome, occurring in a wide range of patients with diverse etiologies, predicting outcome after the onset of ARF is difficult. However information about mortality predictors was from particularly intensive care unit (ICU) $(3,7)$ patients and/or dialysis required patients $(15,17)$. According to our knowledge there was no study that APACHE III scoring system is used as a prognostic factor and involved all ARF patients. So newer prognostic factors, evaluation score and especially regional characteristics of the ARF patients need to be investigated in much more studies.

Therefore in this study, we used APACHE III scoring system as a prognostic factor in all patients with ARF (not only dialyzed or ICU patients), to identify predictors of mortality and to investigate the relationship among these parameters in ARF patients.

\section{Materials and Methods}

\section{Study cohort}

This is a prospective observational cohort study. In this study we wanted to investigate the predictors of mortality in patients with ARF. Therefore, one hundred fifteen patients diagnosed with ARF at Suleyman Demirel University Research Hospital from November 1998 to May 2003 were assessed according to their histories, clinical features, laboratory parameters and outcomes. Fifteen-year-old or older patients were included to the study and patients with chronic kidney disease, renal transplantation and previous dialysis history were excluded.

ARF was defined as an increase in serum creatinine level of at least $0.5 \mathrm{mg} / \mathrm{dL}$ occurring over a 48 -hour period in a patient without renal disease history and with baseline serum creatinine $<1.5 \mathrm{mg} / \mathrm{dL}$. ARF etiologies were diagnosed based on clinical signs, physical examination and laboratory parameters. Acute tubular necrosis (ATN) diagnosis was made when no improvement in renal function was observed after correction of possible prerenal causes as well as by urinary examination and renal ultrasonography. Moreover, re- 
nal biopsy could be performed in patients with unknown etiologies. However, there was no patient in whom renal biopsy was necessary.

The study was approved by the local Ethical Committee and written informed consent was obtained from all participants or relatives.

\section{Patient characteristics and measurements}

All patient's demographics and clinical characteristics, and the cause of ARF were recorded at the first nephrology consultation and patients were followed from the time of initial nephrology consultation to the hospital discharge. Patient's hydration status and daily fluid requirement was assessed according to blood and jugular venous pressure, lower extremity pitting edema and daily weights during follow-up period. Co-morbid conditions were classified in seven parts; diabetes mellitus, hypertension, coronary artery disease, sepsis, pulmonary disease, cerebrovascular disease and liver disease. In addition, presence and the total number of all were recorded.

Blood samples for blood urea nitrogen (BUN), serum creatinine and serum albumin were drawn at the first nephrology consultation time and all measurements were performed by standard clinical laboratory methods in central laboratories. Daily renal function tests, dialysis requirement data, outcomes of the patients and urine output (urine output of $<400 \mathrm{~mL} /$ day defined as oliguria) were recorded during the follow-up period. The primary end-point of the study was hospital mortality.

\section{Dialysis requirement}

All patients were assessed for dialysis therapy indications during the follow-up period. The indications for dialysis included; hyperkalemia (serum potassium $>7 \mathrm{mmol} / \mathrm{L}$ ) resistant to conservative therapy, presence of uremic encephalopathy, metabolic acidosis (serum bicarbonate $<15$ $\mathrm{mEq} / \mathrm{L}$ ) and volume overload with pulmonary edema inadequately controlled with diuretic therapy. Standard hemodialysis schedule was four hours for every session. Conventional bicarbonate hemodialysis was performed, with an ultrafiltration-controlled delivery system, with Hemodialysis Baxter SPS 1550 hemodialysis machine and standard heparin as the anticoagulant agent. The heparinfree hemodialysis method was used if it was necessary. The biocompatible membranes, low flux polysulfone (F5, F6 Fresenius, Bad Homburg, Germany) were used.

\section{APACHE III scoring and Outcome Measurements}

APACHE III scoring system was used as clinical evaluation score. Calculation of the APACHE III score was done as defined before [11] at the first nephrology consultation by the same doctor for each patient. Survival was assessed as discharge alive from the hospital. Survivor and non-survivor patients were compared for ARF etiologies, clinical characteristics, laboratory parameters, APACHE III scores and presence of oliguria to determine the factors effect on survival.

\section{Statistical Analysis}

Parametric values were expressed as means \pm SD. P < 0.05 was considered statistically significant. Student's $t$-test and Mann-Whitney- $U$ test were used to compare two groups. Spearman's $r_{\mathrm{s}}$ correlation test was used to obtain the correlation between two parameters. Univariate analysis of non-parametric factors between survivors and nonsurvivors was accomplished using the chi-square $\left(\chi^{2}\right)$ test. Multiple logistic regression test was used to determine the effects of parameters on mortality rate. We used the chisquare $\chi^{2}$ test of trend to analyze mortality rates associated with APACHE III scores among all patients. Cumulative survival curves were constructed as time to died plots by Kaplan-Meier survival methods, and differences between the curves were tested for significance by the Log-Rank statistics.

\section{Results}

\section{Patient characteristics}

One hundred fifteen patients aged 15 to 90 years, diagnosed with ARF in our tertiary hospital from November 1998 to May 2003, enrolled in this study. Sixty six (58\%) of the patients were men and forty nine (42\%) were women. The mean age of the patients was $64.5 \pm 14.8$ years and most of the patients were older than 60 years. The most common mechanism of ARF was ischemic acute tubular necrosis (51\%). Except two patients, other all patients had at least one co-morbid condition and the rate of the patients with $\geq$ two co-morbid conditions was $46 \%$. The mean APACHE III score was $64.5 \pm 22.1$ (minimum 26 and maximum 145) and BUN and creatinine levels were $75.9 \pm 3.1$ and $4.36 \pm 0.28 \mathrm{mg} / \mathrm{dL}$ respectively at the time of nephrology consultation.

Most of the patients were non-oliguric and oliguria established in only 43 patients $(37 \%)$. The oliguria duration was one day in fourteen patients, two days in thirteen patients, three days in four patients, four days in five patients, five days in two patients, six days in two patients, seven days in one patient and thirteen days in one patient. Twenty-four patients (20\%) received renal replacement therapy (22 HD, 2 PD). Thirty patients died during the following period and the mortality rate was $26 \%$. Demographic and clinical characteristics of the patients are shown in Tab. 1.

\section{Overall survival}

The primary end-point was hospital mortality and 30 (26\%) patients died during the follow-up period in the present study. The causes of mortality are sepsis in seven patients, malignancy in seven patients, cerebrovasculer accident in six patients, end-stage congestive heart failure in five patients, decompansated chronic obstructive lung disease in three patients, gastrointestinal bleeding in one patient and multi-trauma in one patient. Both survivors $(n=$ $85)$ and non-survivors $(n=30)$ were compared for demo- 
Tab. 1: Characteristics of the patients.

\begin{tabular}{|c|c|c|c|c|}
\hline & $\begin{array}{c}\text { Total } \\
(n=115)\end{array}$ & $\begin{array}{c}\text { Survivors } \\
(n=85)\end{array}$ & $\begin{array}{c}\text { Non-survivors } \\
(n=30)\end{array}$ & $P^{*}$ \\
\hline Age (years) & $64.5 \pm 14.8$ & $64.8 \pm 15.1$ & $63.6 \pm 14.0$ & 0.70 \\
\hline Male/Female (n) & $66 / 49$ & $50 / 35$ & $16 / 14$ & 0.60 \\
\hline Mechanical ventilation $(\mathrm{n})$ & 13 & $2(2 \%)$ & $11(36 \%)$ & 0.001 \\
\hline Co-morbid condition $(n)$ & $2.34 \pm 0.10$ & $2.11 \pm 0.99$ & $3.00 \pm 1.08$ & 0.001 \\
\hline Patient with $\geq 2$ co-morbid conditions $(n)$ & 53 & $33(39 \%)$ & $20(66 \%)$ & 0.009 \\
\hline \multicolumn{5}{|l|}{ Etiology of $A R F(n)$} \\
\hline Ischemic ATN & $45(39 \%)$ & $22(25 \%)$ & $23(76 \%)$ & 0.001 \\
\hline Acute interstitial nephritis & $4(3 \%)$ & $3(3 \%)$ & $1(3 \%)$ & 1.0 \\
\hline Nephrotoxic ATN & $17(14 \%)$ & $13(15 \%)$ & $4(13 \%)$ & 0.96 \\
\hline Congestive heart failure & $10(8 \%)$ & $9(10 \%)$ & $1(3 \%)$ & 0.45 \\
\hline Dehydration & $29(25 \%)$ & $28(32 \%)$ & $1(3 \%)$ & 0.001 \\
\hline Acute glomerulonephritis & $2(2 \%)$ & $2(3 \%)$ & 0 & 1.0 \\
\hline Systemic vasculitis & $3(2 \%)$ & $3(5 \%)$ & 0 & 0.56 \\
\hline Postrenal & $5(4 \%)$ & $5(6 \%)$ & 0 & 0.32 \\
\hline Dialysis received patients $(n)$ & 24 & $18(21 \%)$ & $6(20 \%)$ & 0.89 \\
\hline Oliguric patient $(n)$ & 42 & $32(37 \%)$ & $10(33 \%)$ & 0.59 \\
\hline APACHE III score * & $60(60-68)$ & $57(55-59)$ & $85(80-96)$ & 0.0006 \\
\hline Serum albumin $(\mathrm{g} / \mathrm{dL})$ & $3.38 \pm 0.77$ & $3.56 \pm 0.75$ & $2.86 \pm 0.57$ & 0.0002 \\
\hline
\end{tabular}

*Comparison of survivors and non-survivors; ${ }^{* *}$ Mean and $95 \%$ confidence interval within parentheses. ATN; acute tubular necrosis, APACHE; Acute Physiology and Chronic Health Evaluation (APACHE) III score. P $<0.05$ was considered statistically significant. Student's $t$-test, Mann-Whitney- $U$ test and chi-square $\left(\chi^{2}\right)$ test were used to compare two groups.

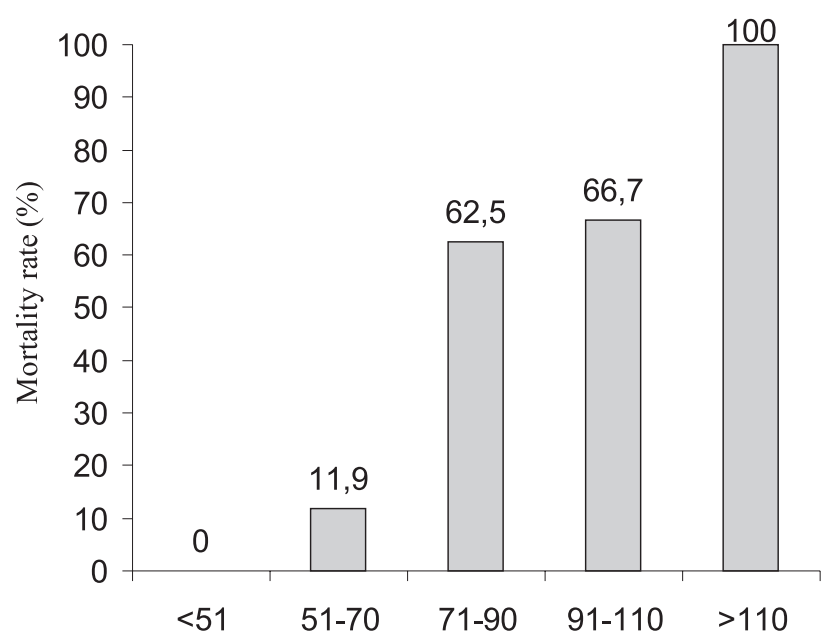

APACHE III score

Fig. 1: The relationship between APACHE III scores and mortality rate.

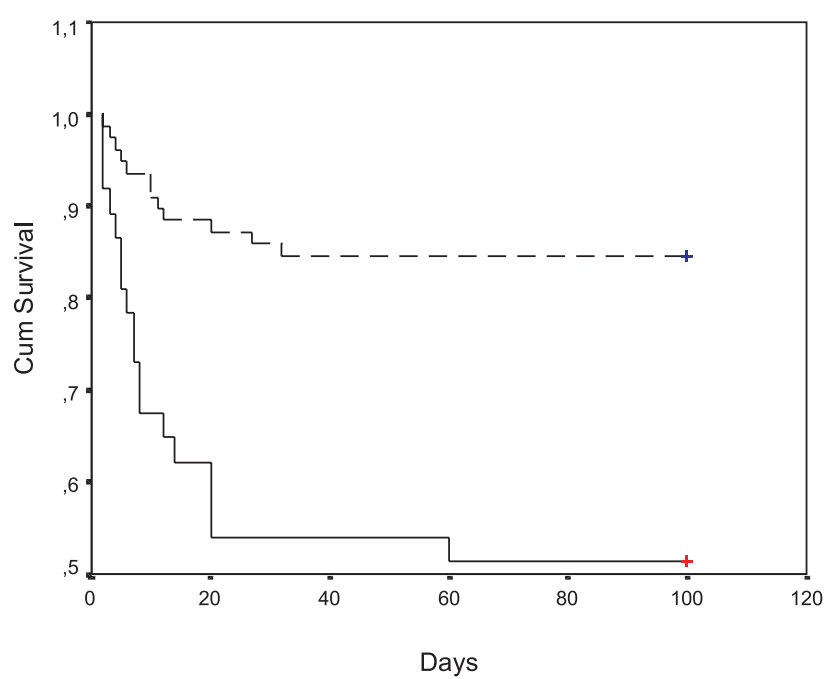

Fig. 2: Cumulative survey graphics in patients with serum albumin $\geq 3 \mathrm{~g} / \mathrm{dL}$ and $<3 \mathrm{~g} / \mathrm{dL}$. Cumulative survival curves were constructed as time to died plots by Kaplan-Meier survival methods, and differences between the curves were tested for significance by the Log-Rank statistics. ---, patients with serum albumin level $\geq 3 \mathrm{~g} / \mathrm{dL}$. , patients with serum albumin level $<3 \mathrm{~g} / \mathrm{dL}$. 
graphic and clinical parameters, laboratory parameters, APACHE III scores, dialysis requirement, oliguria rate and presence of co-morbid conditions. Both groups were similar for age, gender, dialysis requirement and oliguria rate. Not surprisingly that mechanical ventilated patient ratio, the rate of patients with $\geq$ two co-morbid conditions and APACHE III score were significantly higher in non-survivors than survivors. In addition, serum albumin level was higher in survivors than non-survivors. Comparison of both groups is shown in Tab. 1.

\section{Predictors of mortality}

There was an evident and significant increase in mortality rate associated with an increase in the APACHE III score (exceed 60), decrease in serum albumin level (serum albumin $\leq 3 \mathrm{~g} / \mathrm{dl}$ ) and presence of co-morbid conditions [Relative risk (RR); 29.6 P =0.0001, RR; 8.6 P = 0.0001 and RR; $10.6 \mathrm{P}=0.006$ respectively]. Increased APACHE III score was found the most powerful predictor of mortality and increase in mortality rate became evident when the APACHE III score exceed 70 (Fig. 1).

Serum albumin level was found higher in survivors compared to non-survivors $(3.56 \pm 0.75 \mathrm{~g} / \mathrm{dL}$ vs $2.86 \pm 0.57$ $\mathrm{g} / \mathrm{dL}$ respectively, $\mathrm{P}=0.0002$ ). In addition, we found that mortality rate increases by the serum albumin level decreases. Such as, mortality rate was found $22 \%$ in patients with serum albumin level between $3-3.9 \mathrm{~g} / \mathrm{dL}$ and by decreasing in serum albumin for each $1 \mathrm{~g} / \mathrm{dL}$, mortality rate increases two times. However, mortality rate was 30 times higher in patients with serum albumin level $<2 \mathrm{~g} / \mathrm{dL}$ than in those with serum albumin level $\geq 4 \mathrm{~g} / \mathrm{dL}$. In addition, when the patients are divided to two groups according to serum albumin level $\geq 3.0 \mathrm{~g} / \mathrm{dL}$ or $<3.0 \mathrm{~g} / \mathrm{dL}$, we found that survival was better in patients with serum albumin level $\geq$ $3.0 \mathrm{~g} / \mathrm{dL}$. Comparison of survival is shown in Fig. 2.

\section{Dialysis requirement}

Twenty-four patients (20\%) required dialysis therapy (twenty-two HD and two PD). Dialyzed and non-dialyzed patients were similar for age, serum albumin levels and APACHE III scores. Serum creatinine level was higher in dialyzed patients than those of non-dialyzed patients (in order to $8.12 \pm 4.26$ versus $4.11 \pm 2.14 \mathrm{mg} / \mathrm{dL} \mathrm{P}=0.0004$ ). Oliguric days were longer in dialysis required patients than those of non-dialysis required group $(2.5 \pm 3.2$ days versus $0.5 \pm 1.0$ days respectively, $\mathrm{P}=0.0004)$. Oliguria rate was higher in dialysis required patients $(45 \%$ and $35 \%$ respectively), but not statistically significant. In addition, mortality rate was similar between oliguric and non-oliguric patients ( $27 \%$ vs $23 \%$ respectively). Six of 24 patients died in dialysis required group, but in non-dialysis required group 24 of 91 patients died. However, mortality rate was similar in both groups ( $25 \%$ versus $26 \%$ ).

\section{Correlations}

We found that higher serum albumin level and lower APACHE III score are the good prognostic factors. So, we investigated the correlation between serum albumin level and APACHE III score. There was a negative correlation between serum albumin level and APACHE III score. By the decreasing in serum albumin level, APACHE III score and also mortality rate increase $\left(\mathrm{r}^{2}=0.21 \mathrm{P}=0.003\right)$.

\section{Discussion}

The main finding of this study is that decreased serum albumin level, increased APACHE III score and presence of co-morbid conditions at the first nephrology consultation time were the independent predictors of outcomes in patients with ARF and APACHE III score was found the most powerful predictor of mortality among these parameters. In this study, prediction power of mortality of these parameters, were found higher than recent published reports $(1,19,21)$. However, despite of recently published report (21) we found that the mortality prediction power of serum albumin level was found higher and serum albumin level attracted attention as alone possible modifiable predictor factor.

This is a single center prospective observational study and the number of patients is limited. However, all patients diagnosed with ARF in our hospital between 1998 and 2003 were included. Recently published reports about predictors of mortality in ARF patients were from particularly ICU patients and reports about out of ICU patients were limited. In this study all patients with ARF were included to the study and were not separated according to ICU patient or not. According to our knowledge, this is the first study that APACHE III scoring system was used as a prognostic factor in the assessment of all ARF patients.

In recent published reports the mortality rate has been reported 50 to $80 \%$ of the patients with ARF $(11,12,24)$. However, total mortality rate was $26 \%$ in present study and similar to Chertow (7) and Clermont's (8) reports. Most of the ICU and most of the mechanical ventilated patients died. In this study all patients diagnosed ARF in our tertiary hospital were included to study protocol. We did not include only ICU patients and dialyzed patients, therefore mortality rate was found lower than the other reports. The reason for low mortality rate could be owing to the small number of ICU patients and mechanical ventilated patients.

Morgera et al (19) reported that age and more than one co-morbid condition before hospitalization were associated with significantly lower post-discharge survival. In present study, survivors and non-survivors were similar for age, but patient ratio, with $\geq$ two co-morbid conditions, was higher in non-survivors. So our results are similar Morgera's report and confirmed the importance of co-morbid conditions on survival in ARF patients.

Different prognostic factors, associated with mortality such as urine output (13), dialysis requirement (17) and clinical evaluation scores $(3,17)$, have been reported in patients with ARF. Liangos et al (17) reported that urine volume was independently associated with hospital mortality 
and hospital mortality was increased by the increasing of urine output in patients with ARF. In present study, mortality rate was similar in both oliguric and non-oliguric patients ( $27 \%$ and $23 \%$ respectively). Daily urine output was not obtained as a predictor of hospital mortality in this study.

Clermont $G$ et al (8) reported that patients with ARF severe enough to require dialysis had a mortality of $57 \%$ and Chen et al (6) reported that in dialysis required patients with ARF the overall mortality was $71 \%$. In present study 24 patients required dialysis, 6 of them died during the study, mortality rate in dialysis required patients was $25 \%$ and also this is a lower rate than above studies.

In the literature, most of the studies assessed the predictors of mortality and the prognostic factors in patients with ARF. APACHE II (6) and III (5), Sequential Organ Failure Assessment (SOFA) (9) and acute tubular necrosisindividual severity index (ATN-ISI) (10) are frequently used for assessment of mortality especially in ICU and with multiple organ dysfunction patients. In this study APACHE III score was used as clinical evaluation score. Chen (6) and Parker (23) also obtained APACHE III score within 24 hours of initiation of dialysis in all patients required dialysis with ARF. However we also used APACHE III score in all ARF patients and this application is an important property of this study. As recent study (5), we also found strong association between APACHE III score and mortality rate. In this study, APACHE III score was significantly higher in survivor group and was the most significant predictor of mortality, when the other predictors, serum albumin level and presence of co-morbid conditions were included. APACHE III score, performed at the first nephrology consult day, predicted the mortality of the patients. In present study, none of the patients died with APACHE III score lower than 50. We found that mortality rate became evident by the APACHE III score exceed 70 . It was 5 times higher in patients with APACHE III score $>70$ than in patients with APACHE III score $<70$.

In the laboratory parameters only serum albumin level was found as a predictor of mortality in ARF patients. Hypoalbuminemia have been reported to be independent predictors of total and cardiovascular mortality in patients with end-stage renal disease (ESRD) $(18,22)$. Hypoalbuminemia was recently reported by Chertow et al (7) as a predictor of mortality with a RR of 0.56 in their patients with acute tubular necrosis. Similarly hypoalbuminemia was reported by Obialo et al (21) as a predictor of mortality with an RR of 3.6 in their patients with ARF. We observed a much higher RR of death. In present study mortality rate was 5 times higher in patients with serum albumin level $<2$ $\mathrm{g} / \mathrm{dL}$, than in patients with serum albumin level 3-3.9 g/dL. However, mortality rate was 30 times higher in patients with serum albumin level $<2 \mathrm{~g} / \mathrm{dL}$, than in patients with serum albumin level $\geq 4 \mathrm{~g} / \mathrm{dL}$. This is also suggested the importance of serum albumin level and effects of serum albumin on mortality rate.
It could be speculated that serum albumin level might fluctuate considerably with hydration. However, blood samples were drawn at the first nephrology consultation and the change in serum albumin level during follow-up period did not considered in present study. This is one of the limitations of this study.

In conclusion, despite of limitations in present study, not only increased APACHE III score and presence of comorbid conditions but also low serum albumin level was found as the predictors of mortality in patients with ARF. However, among these prognostic parameters only serum albumin level is seen as modifiable prognostic factor. Therefore, further studies are necessary to determine the causes of hypoalbuminemia in patients with ARF and the effect of it's effective treatment on patients outcome.

\section{References}

1. Batista PB, Cendorogolo Neto $\mathrm{M}$ et al. Evaluation of prognostic indexes in critical acute renal failure patients. Ren Fail. 2004;26:545-52.

2. Biesenbach G, Zazgornik J, Kaiser W et al. Improvement in prognosis of patients with acute renal failure over a period of 15 years: An analysis of 710 cases in a dialysis center. Am J Nephrol 1992;12:319-25

3. Brivet FG, Kleinknecht DJ, Loirat P, Landais PJ. Acute renal failure in intensive care units-causes, outcome, and prognostic factors of hospital mortality; a prospective, multicenter study. French Study Group on Acute Renal Failure. Crit Care Med 1996;24:192-8.

4. Bullock ML, Umen AJ, Finkelstein M, Keane WF. The assessment of risk factors in 462 patients with acute renal failure. Am J Kidney Dis 1985;5:97-103.

5. Chen YC, Hsu HH, Chen CY et al. Integration of APACHE II and III scoring systems in extremely high risk patients with acute renal failure treated by dialysis. Ren Fail. 2002;24:285-96.

6. Chen YC, Hsu HH, Kao KC et al. Outcomes and APACHE II predictions for critically ill patients with acute renal failure requiring dialysis. Ren Fail 2001;23: 61-70.

7. Chertow GM, Lazarus JM, Paganini EP et al. Predictors of mortality and the provision of dialysis in patients with acute tubular necrosis. The Auriculin Anaritide Acute Renal Failure Study Group. J Am Soc Nephrol 1998;9:692-8.

8. Clermont G, Acker CG, Angus DC et al. Renal failure in the ICU: comparison of the impact of acute renal failure and end-stage renal disease on ICU outcomes. Kidney Int. 2002;62:986-96.

9. Dara SI, Afessa B, Bajwa AA, Albright RC. Outcome of patients with end-stage renal disease admitted to the intensive care unit. Mayo Clin Proc. 2004;79: 1385-90.

10. D'Avila DO, Cendoroglo Neto M, dos Santos OF et al. Acute renal failure needing dialysis in the intensive care unit and prognostic scores. Ren Fail. 2004;26: 59-68.

11. Heering $\mathrm{P}$, Ivens $\mathrm{K}$, Thumer $\mathrm{O}$ et al. The use of different buffers during continuous hemofiltration in critically ill patients with acute renal failure. Intensive Care Med. 1999 Nov;25:1244-51.

12. Heering P, Morgera S, Schmitz FJ et al. Cytokine removal and cardiovascular hemodynamics in septic patients with continuous venovenous hemofiltration. Intensive Care Med. 1997;23:288-96.

13. Hou SH, Bushinsky DA, Wish JB et al. Hospital-acquired renal insufficiency A prospective study. Am J Med 1983;74:243-8.

14. Knaus WA, Wagner DP, Draper EA et al. The APACHE prognostic system; risk prediction of hospital mortality. Chest 1991;100:1619-36.

15. Leblanc M, Tapolyai M, Paganini EP. What dialysis dose should be provided in acute renal failure? A review. Adv Ren Replace Ther 1995;2:255-364.

16. Levy EM, Viscoli CM, Horwitz RI. The effect acute renal failure on mortality. A cohort analysis. JAMA 1996;275:1489-94.

17. Liangos O, Rao M, Balakrishnan VS et al. Relationship of urine output to dialysis initiation and mortality in acute renal failure. Nephron Clin Pract 2005;99: 56-60.

18. Lowrie EG, Lew NL. Death risk in hemodialysis patients: the predictive value of commonly measured variables and an evaluation of death rate differences between facilities. Am J Kidney Dis 1990;15:458-82.

19. Morgera S, Kraft AK, Siebert G et al. Long-term outcomes in acute renal failure patients treated with continuous renal replacement therapies. Am J Kidney Dis 2002;40:275-9. 
20. Nolan CR, Anderson RJ. Hospital-acquired acute renal failure. J Am Soc Nephrol 1998;9:710-18

21. Obialo CI, Okonofua EC, Nzerue MC et al. Role of hypoalbuminemia and hypocholesterolemia as copredictors of mortality in acute renal failure. Kidney Int 1999;56:1058-63.

22. Owen WF Jr, Lew NL, Liu $\mathrm{Y}$ et al. The urea reduction ratio and serum albumin concentration as predictors of mortality in patients undergoing hemodialysis. N Engl J Med 1993;329:1001-6.
23. Parker RA, Himmelfarb J, Tolkoff-Rubin $\mathrm{N}$ et al. Prognosis of patients with acute renal failure requiring dialysis:results of a multicenter study. Am J Kidney Dis. 1998:32:432-43.

24. Ronco C, Bellomo R, Homel P et al. Effects of different doses in continuous veno-venous haemofiltration on outcomes of acute renal failure: a prospective randomised trial. Lancet. $2000 \mathrm{Jul} ; 356: 26-30$.

25. Shusterman N, Strom BL, Murray TG et al. Risk factors and outcome of hospitalacquired acute renal failure. Clin. epidemiologic study. Am J Med 1987;83:65-71.

Submitted April 2006

Accepted August 2006

Dr. Mehmet Tugrul SEZER, Suleyman Demirel Universitesi, Tip Fakultesi Ic Hast. Nefroloji ABD,

Nefroloji BD, Cunur Isparta, Turkey, e-mail: tugrul@med.sdu.edu.tr 